\title{
The Second-Quantized Theory of Spin- $\frac{1}{2}$ Particles in the Nonrelativistic Limit* $\dagger$
}

\author{
RICHARD K. OSBORN $\ddagger$ \\ Case Institute of Technology, Cleveland, Ohio
}

(Received September 17, 1951)

\begin{abstract}
The second-quantized Dirac Hamiltonian for free electrons is transformed by a canonical transformation to a representation in which the positive and negative energy wave operators are separately represented by two-component operators. The transformation employed is the second-quantized analog of the one derived by Foldy and Wouthuysen in their discussion of the one-particle Dirac theory and its nonrelativistic limit. This transformation is then applied to the wave operators and the Hamiltonian in the secondquantized, charge-conjugate formalism for Dirac particles. The wave operators for positrons and electrons become linearly-independent two-component operators, and the Hamiltonian separates into an electron and a positron part, each of which contains only the corresponding two-component wave operators.

It is also shown that by means of an appropriate, readily determinable sequence of canonical transformations, Hamiltonians for fields of spin- $\frac{1}{2}$ particles interacting via intermediary fields can also be reduced to nonrelativistic form. This is accomplished by transforming the Hamiltonian to a representation in which it is exhibited effectively as a series expansion in powers of the Compton wavelength of the spin $-\frac{1}{2}$ particle. Illustration of the method is provided by detailed examination of the case of nucleons interacting via the pseudoscalar meson field.
\end{abstract}

\section{INTRODUCTION}

$T$ HEORIES of elementary particles and their interactions must at the present time be formulated in very general terms, since the mathematical difficulties of solution and the experimental difficulties of verification of detailed theories have as yet not been surmounted. The general requirements imposed upon theories of elementary particles and their interactions in a first-quantized formalism are usually that the equations describing the system shall be invariant under the group of Lorentz and gauge transformations, and that the function containing the physical description of the particle shall have certain special transformation properties corresponding to the known, or presumably known, spin of the particle.

However, because of the fact that the number of interacting particles is generally not constant throughout the course of the interaction, the mathematical description of such interacting systems is most easily obtained in the second-quantized formalism of field theory in which the state function becomes a vector in quantum number space instead of coordinate space, and the old one-particle functions whose transformation properties were prescribed by the ascribed spin of the particles go over into suitably transforming creation and destruction operators. The Lorentz and gauge invariance requirements are retained in the field formalism and are usually accomplished by first assuming that the proper conjugate variables, equations of motion, and Hamiltonians are derivable from some suitable Lagrangian regarded as a functional of the appropriate creation and destruction dynamical variables, and then prescribing that the Lagrangian shall be Lorentz and

\footnotetext{
* This research was supported in part by the AEC.

$\uparrow$ From a thesis submitted to Case Institute of Technology in partial fulfillment of the requirements for the degree of Doctor of Philosophy.

$\ddagger$ Now at Oak Ridge National Laboratory, Oak Ridge, Tennessee.
}

gauge invariant. Starting from this point of view, the commutation rules obeyed by the canonical variables of which the Hamiltonian is a function must be postulated, and the above invariance properties are required of them also.

But, though the most satisfactory mathematical approach to the problem of elementary particles and their interactions is accomplished in the secondquantized field theoretical formalism, there are many practical problems in which a nonrelativistic description of the interacting systems is adequate. The question arises immediately, therefore, relative to consideration of such problems, as to how, in a simple and rigorously satisfying way, the initial relativistically covariant formulation shall be reduced to a nonrelativistic form, which for purposes of calculation is generally simpler to deal with.

This question was answered in the one-particle, or first-quantized, formalism by a method derived by Foldy and Wouthuysen. ${ }^{1}$ They pointed out that this question is especially obscure when particles with $\frac{1}{2}$-integral spin are involved, for in that event many of the dynamical variables occuring in the relativistic formulation have no obvious analog in the corresponding nonrelativistic (Pauli) formalism. The nonrelativistic system of spin- $\frac{1}{2}$ particles is described in terms of two-component wave functions, whereas the relativistic system is usually described in terms of fourcomponent wave functions, and the Hamiltonian generally contains odd operators which couple the upper and lower components of these functions.

If, however, it were possible to transform the Hamiltonian from the usual representation in which it contains odd operators to one in which it contains no odd operators, by means of one or more canonical trans-

${ }^{1}$ L. L. Foldy and S. A. Wouthuysen, Phys. Rev. 78, 29 (1950). Some of the results obtained in the paper relative to noninteracting particles have been previously obtained by Foldy and Wouthuysen in unpublished calculations. 
formations, then it would be possible to describe the system by means of two unconnected sets of two-component wave functions. In this representation, if the transformation can be accomplished exactly, the nonrelativistic approximation can be obtained directly simply by stipulating that $p / m c \ll 1$. Transformations accomplishing the desired change of representation in the first-quantized formalism were deduced by Foldy and Wouthuysen for the free Dirac particle and the Dirac particle interacting with the electromagnetic field.

The purpose of this paper is to generalize this method so that it may be applied in the second-quantized formalism. In this formalism the spinor wave functions go over into four-component creation and destruction operators. Thus, when the Hamiltonian has been freed of odd Dirac operators by appropriate canonical transformations, it will be possible to decompose the fourcomponent spinor operators into two sets of two-component operators, one set creating and destroying only positive energy states of spin- $\frac{1}{2}$ particles, the other set creating and destroying only negative energy states.

\section{THE TRANSFORMATION IN THE CASE OF NO INTERACTION}

The generalizations required for the case of free Dirac fields are fairly obvious, and the results obtained are, in the main, simply a second-quantized transcription of the results in the one-particle formalism. Consequently only a summary of the main points of procedure and results will be presented here.

The Hamiltonian in the usual representation,

$$
H=\int \psi^{*}(\boldsymbol{\alpha} \cdot \mathbf{p}+\beta m) \psi d^{3} x
$$

is transformed by a unitary transformation

$$
\exp (i S)=\exp \left[-\frac{\mathrm{i}}{2} \int \psi^{*} \beta \frac{\boldsymbol{\alpha} \cdot \mathbf{p}}{p} \tan ^{-1}\left(\frac{\mathbf{p}}{m}\right) \psi d^{3} x\right],
$$

to a representation in which it contains no odd operators, i.e.,

$$
H^{\prime}=e^{i S} H e^{-i S}=\int \psi^{*} \beta\left(p^{2}+m^{2}\right)^{\frac{1}{2}} \psi d^{3} x .
$$

The proof of the correctness of Eq. (1) consists primarily in determining how the wave operators $\psi$ transform since

$$
H^{\prime}=\int e^{i S} \psi^{*} e^{-i S}(\boldsymbol{\alpha} \cdot \mathbf{p}+\beta m) e^{i S} \psi e^{-i S} d^{3} x .
$$

Thus, if one expands the transformed operator into the usual series of commutators

$$
\psi^{\prime}=e^{i S} \psi e^{-i S}=\sum_{n=0}^{\infty} \frac{i^{n}}{n !}[S, \psi]^{n},
$$

one finds that by inductive arguments the $n$th order commutator may be explicitly evaluated and the series summed. The result may be most conveniently expressed by the equation

where

$$
\psi^{\prime}=U \psi,
$$

Then

$$
U=(E+m-\beta \boldsymbol{\alpha} \cdot \mathbf{p}) /[2 E(E+m)]^{\frac{1}{2}} .
$$

$$
U^{-1}=(E+m+\beta \boldsymbol{\alpha} \cdot \mathbf{p}) /[2 E(E+m)]^{\frac{1}{2}},
$$

and it is easily verified that

and

$$
U U^{-1}=1 \text {, }
$$

$$
U^{-1}(\boldsymbol{\alpha} \cdot \mathbf{p}+\beta m) U=\beta\left(p^{2}+m^{2}\right)^{\frac{2}{2}} .
$$

In the representation in which $H$ is free of odd operators, the four-component wave operators $\psi$ decompose into two sets of two-component operators containing creation and destruction operators for either positive or negative energy states separately. One can also show that the momentum-space eigenfunctions $u(p, s)$ defined by $^{2}$

$$
\psi=\frac{1}{(2 \pi)^{\frac{3}{2}}} \sum_{s} \int_{p} a(p, s) e^{i \mathrm{p} \cdot \mathbf{x}} u(p, s) d^{3} p
$$

take on a particularly simple form in the new representation. Specifically, the functions $u$ transform according to

$$
u^{ \pm^{\prime}}=\left(\frac{2 E}{E+m}\right)^{\frac{1}{2}}\left(\frac{1 \pm \beta}{2}\right) \Lambda^{ \pm}(p) u(p, s)
$$

where

$$
E=\left(m^{2}+p^{2}\right)^{\frac{1}{2}}, \quad \Lambda^{ \pm}(p)=[E \pm(\boldsymbol{\alpha} \cdot \mathbf{p}+\beta m)] / 2 E,
$$

and, s a consequence, ${ }^{3}$

$$
\begin{aligned}
& u^{+^{\prime}}(p, 1)=\{1,0,0,0\}, \\
& u^{+\prime}(p, 2)=\{0,1,0,0\}, \\
& u^{-\prime}(p, 3)=\{0,0,1,0\}, \\
& u^{-\prime}(p, 4)=\{0,0,0,1\} .
\end{aligned}
$$

A transformation to remove the odd operators from the Hamiltonian for noninteracting spin- $-\frac{1}{2}$ particles in the charge-conjugate formalism was deduced, and the Hamiltonian in the new representation assumed a form that one would intuitively expect. The new Hamiltonian is reducible to a sum of three terms, two of which are functions of either two-component positron creation and destruction operators only, or two-component electron operators only. The third term is an infinite constant which arises as a consequence of the fact that the system has been transformed from a charge conjugate hole theory representation, in which the vacuum corresponds to a state of infinite negative energy, to a representation in which the vacuum cor-

\footnotetext{
${ }^{2}$ L. I. Schiff, Quantum Mechanics (McGraw-Hill Book Company, Inc., New York, 1949), p. 351.

${ }_{3} \mathrm{~W}$. Heitler, The Quantum Theory of Radiation (Oxford University Press, London, 1949), second edition, p. 86.
} 
responds to a state of zero energy. Thus, by the transformation, the eigenvalues of the energy operator have been shifted by an infinite amount corresponding to the "defining away" of the negative energy states. This infinite constant is in no sense a self-energy term.

\section{THE TRANSFORMATION FOR THE CASES IN WHICH INTERACTIONS ARE CONSIDERED}

When fields representing spin- $\frac{1}{2}$ particles interact with other fields, it no longer appears possible to go over to a representation in which the Hamiltonian is free of odd operators and in which the spinor operators have a two-component description, by means of a single canonical transformation. Foldy and Wouthuysen ${ }^{1}$ showed, however, that in the one-particle formalism, the Hamiltonian for the interaction of a spin- $\frac{1}{2}$ particle and a given external field could be freed of odd operators to any desired order in the parameters, $\left(\hbar / m c^{2}\right) \partial / \partial t$ and $(\hbar / m c) \nabla$. The essence of their method consisted in obtaining the Hamiltonian in the form of a power series in these expansion parameters by a succession of readily determinable, unique canonical transformations. An investigation of the conditions under which this expansion is presumably physically significant (that is, that the field variables do not vary appreciably over spatial dimensions of the order of magnitude of the Compton wavelengths of the particles, or over time intervals of the order of the time required for light to travel a Compton wavelength for the particles) emphasizes the fact that the end results of the transformation procedures under consideration are indeed a reduction of the Hamiltonian to nonrelativistic form.

\section{A. Interactions of Two Spin- $\frac{1}{2}$ Particles}

\section{Different Particles}

The problem of removing the odd operators from the Hamiltonian in the cases in which the Dirac particles are presumed to interact with given external fields proceeds in strict analogy to the same problem in the one-particle formalism.

If, however, one considers cases in which two or more Dirac particles interact through the intermediation of another field, whether the Dirac particles be representable by different fields as in the case of neutrons and protons interacting via the meson field, or representable by a single field as in the case of two electrons interacting via the electromagnetic field, one encounters types of odd terms which at first sight present apparent difficulties in the application of the above method. The difficulty arises as a consequence of the generation of terms which are essentially products of two or more factors, each separately odd. In general these terms are neither even nor removable in the form in which they arise by further canonical transformations. The difficulty is merely apparent, however, and resolves itself after an examination of these terms in the light of their physical significance.
Though there is no difficulty in principle, it is too laborious to carry through the reduction of the Hamiltonian for arbitrarily interacting systems to a form free of odd operators to a given order in inverse powers of the masses. Consequently, the case of nucleonic interactions via the pseudoscalar meson field with both pseudoscalar and pseudovector coupling will be selected for detailed consideration. This example is chosen because of its current practical importance; because it satisfactorily illustrates the nature, source, and resolution of the difficulties commented upon above; and because it provides a statement of the equivalence theorem for pseudoscalar and pseudovector coupling in the nonrelativistic limit. To further reduce the length of the calculations involved, another restriction is made to the case of charged mesons only, and electromagnetic interactions will be omitted.

The Schrödinger equation for the system under consideration is

$$
H \Phi=i \partial \Phi / \partial t,
$$

where $\Phi$ is the time-dependent state function and $H=\int \mathcal{F C} d^{3} x$ is the time-independent Hamiltonian. The Hamiltonian density $\mathfrak{H}$ is obtainable most directly by derivation from a Lagrangian density $\mathscr{L}$ by the prescription

$$
\mathfrak{H}=-\mathfrak{L}-\frac{\partial \mathfrak{L}}{\partial\left(\partial \varphi_{A} / \partial x_{\mu}\right)} n_{\mu} n_{\nu} \frac{\partial \varphi_{A}}{\partial x_{\nu}} .
$$

The $\varphi_{A}$ are the various fields comprising the system and $\mathbf{n}$ is a unit four-vector normal to the space-time surface at the point $x$. Derivatives normal to the spacetime surface may be eliminated from $\mathfrak{H C}$ by making use of the definitions of the momenta

$$
P_{A}=-\frac{\partial \mathscr{L}}{\partial\left(\partial \varphi_{A} / \partial x_{\mu}\right)} n_{\mu}
$$

and then the noncovariant canonical formalism convenient for the discussion that follows is obtainable immediately by specializing to a flat space-like surface for which $n=(0,0,0, i)$. For the case to be discussed presently

$$
\begin{aligned}
\mathscr{L}=-\bar{\Psi}\left(\gamma^{\mu} \frac{\partial \Psi}{\partial x_{\mu}}+m \Psi\right)-\left(\frac{\partial \phi^{*}}{\partial x_{\mu}} \frac{\partial \phi}{\partial x_{\mu}}+\mu^{2} \phi^{*} \phi\right) \\
-\left(R \phi^{*}+R^{*} \phi\right)-\left(\Gamma_{\mu} \frac{\partial \phi}{\partial x_{\mu}}+\Gamma_{\mu} \frac{\partial \phi^{*}}{\partial x_{\mu}}\right),
\end{aligned}
$$

and the final form of $\mathcal{H}$ with which we have here to deal is

$$
\begin{gathered}
\mathcal{F}=\mathcal{F}_{m 0}+\Psi^{*} \boldsymbol{\alpha} \cdot \mathbf{p} \Psi+\Psi^{*} \beta m \Psi+i f \Psi^{*} \beta \gamma^{5}\left(\tau_{-} \phi+\tau_{+} \phi^{*}\right) \Psi \\
-(g / \mu) \Psi^{*} \boldsymbol{\sigma} \cdot\left(\tau_{-} \boldsymbol{\nabla} \phi+\tau_{+} \boldsymbol{\nabla} \phi^{*}\right) \Psi \\
+(g / \mu) \Psi^{*} \gamma^{5}\left(\tau_{-} \pi^{*}+\tau_{+} \pi\right) \Psi \\
+\frac{1}{2}(g / \mu)^{2}\left[\Psi^{*} \gamma^{5} \tau_{-} \Psi \Psi^{*} \gamma^{5} \tau_{+} \Psi\right. \\
\left.+\quad+\Psi^{*} \gamma^{5} \tau_{+} \Psi \Psi^{*} \gamma^{5} \tau_{-} \Psi\right],
\end{gathered}
$$


where

$$
\begin{aligned}
R & =i f \Psi \gamma^{5} \tau_{+} \Psi \\
\Gamma_{\mu} & =(i g / \mu) \bar{\Psi} \gamma^{\mu} \gamma^{5} \tau_{+} \Psi \\
\mathcal{F}_{m 0} & =\pi^{*} \pi+\nabla \phi^{*} \cdot \nabla \phi+\mu^{2} \phi^{*} \phi
\end{aligned}
$$

The constants $\mu, f$, and $g$ are the meson mass and the pseudoscalar and pseudovector coupling constants, respectively. The nucleon wave operators $\Psi$ and $\bar{\Psi}=\Psi^{*} \beta$ are eight-component column and row matrix operators $\left\{\psi_{a}, \psi_{b}\right\}$ where $\left\{\psi_{a}, 0\right\}$ represents the neutron and $\left\{0, \psi_{b}\right\}$ the proton;

$$
\tau_{+}=\frac{1}{2}\left(\tau_{x}+i \tau_{y}\right) \text { and } \tau_{-}=\frac{1}{2}\left(\tau_{x}-i \tau_{y}\right)
$$

are the operators that accomplish the change in charge state of the nucleons as a consequence of the interaction with the meson field; and the Dirac matrices $\gamma^{\mu}=(-i \beta \boldsymbol{\alpha}, \beta)$ have in this representation the form

$$
\alpha=\left(\begin{array}{ll}
\alpha & 0 \\
0 & \alpha
\end{array}\right) \text {. }
$$

The quantities $\phi$ and $\phi^{*}$ are the meson field operators, and $\pi$ and $\pi^{*}$ are their conjugate momenta. The commutation rules obeyed by the field operators are

$$
\begin{aligned}
{\left[\phi^{*}, \pi^{* \prime}\right]_{-} } & =\left[\phi, \pi^{\prime}\right]_{-}=i \delta\left(\mathbf{x}-\mathbf{x}^{\prime}\right), \\
{\left[\Psi_{i}^{*}, \Psi_{j}^{\prime}\right]_{+} } & =\delta_{i j} \delta\left(\mathbf{x}-\mathbf{x}^{\prime}\right) .
\end{aligned}
$$

The procedure that follows is prescribed by the requirement that odd operators be removed from the Hamiltonian to the order $\left(1 / m^{2}\right)$. Further, explicit cognizance will be taken of the fact that $g / \mu \sim(f / m)$. Write

$$
H=\sum_{i=1}^{7} H_{i}
$$

where

$$
H_{i}=\int \mathcal{H}_{i} d^{3} x
$$

and where

$$
\begin{aligned}
& \mathfrak{H}_{1}=\Psi^{*} \beta m \Psi \\
& \mathfrak{F}_{2}=\mathfrak{H C}_{m 0} \text {, } \\
& \mathfrak{H}_{3}=\Psi^{*} \boldsymbol{\alpha} \cdot \mathbf{p} \Psi \\
& \mathfrak{H}_{4}=i f \Psi^{*} \beta \gamma^{5}\left(\tau_{\llcorner} \phi+\tau_{+} \phi^{*}\right) \Psi \text {, } \\
& \mathfrak{H}_{5}=(g / \mu) \Psi^{*} \gamma^{5}\left(\tau_{-} \pi^{*}+\tau_{+} \pi\right) \Psi \text {, } \\
& \mathfrak{H}_{6}=-(g / \mu) \Psi^{*} \boldsymbol{\sigma} \cdot\left(\tau_{-} \boldsymbol{\nabla} \phi+\tau_{+} \boldsymbol{\nabla} \phi^{*}\right) \Psi \text {, } \\
& \mathfrak{H C}_{7}=\frac{1}{2}(g / \mu)^{2}\left[\Psi^{*} \gamma^{0} \tau_{-} \Psi \Psi^{*} \gamma^{5} \tau_{+} \Psi+\Psi^{*} \gamma^{5} \tau_{+} \Psi \Psi^{*} \gamma^{5} \tau_{-} \Psi\right] \\
& =\frac{1}{2}(g / \mu)^{2}(\mathrm{DI}) \text {, }
\end{aligned}
$$

where (DI) stands for the direct interaction.

The lowest order odd operators, to be removed first, are the terms $\Psi^{*} \boldsymbol{\alpha} \cdot \mathbf{p} \Psi$, if $\Psi^{*} \beta \gamma^{5}\left(\tau_{-} \phi+\tau_{+} \phi^{*}\right) \Psi$, and $(g / \mu) \Psi^{*} \gamma^{5}\left(\tau_{-} \pi^{*}+\tau_{+} \pi\right) \Psi$. The transformation required is $e^{i T}$, where

$$
\begin{aligned}
& T=-(i / 2 m) \int \Psi^{*}\left[\beta \boldsymbol{\alpha} \cdot \mathbf{p}+i f \gamma^{5}\left(\tau_{-} \phi+\tau_{+} \phi^{*}\right]\right. \\
& \left.+(g / u) \beta \gamma^{5}\left(\tau_{-} \pi^{*}+\tau_{+} \pi\right)\right] \Psi d^{3} x . \\
& \mathfrak{H C}^{\prime}=e^{i T} \mathfrak{H C} e^{-i T}=\mathfrak{H C}+i[T, \mathfrak{F C}]+\frac{1}{2} i^{2}[T, \mathfrak{H C}]^{2} \\
& +\frac{1}{6} i^{3}\left[T, \mathfrak{F}_{1}\right]^{3} \text { to } \sim 1 / m^{2} \text {. }
\end{aligned}
$$

The commutators may be evaluated by straightforward calculation, the labor being considerably reduced by employment of the relation

$$
\begin{aligned}
& {\left[\int \Psi^{*} A \Psi a d^{3} x, \Psi^{*} B \Psi b\right]} \\
& \quad=\int\left[a(x), b\left(x^{\prime}\right)\right] \Psi^{*}(x) A \Psi(x) \Psi^{*}\left(x^{\prime}\right) B \Psi\left(x^{\prime}\right) d^{3} x \\
& +b(x) a(x) \Psi^{*}(x)[A, B] \Psi(x),
\end{aligned}
$$

where $a(x)$ and $b(x)$ are operators commuting with $\Psi$ and $\Psi^{*}$, but such that $\left[a, b^{\prime}\right]$ is proportional to $\delta\left(\mathbf{x}-\mathbf{x}^{\prime}\right)$, and $A$ and $B$ are arbitrary functions of $\nabla, \beta, \alpha, \gamma^{5}, \tau_{+}$, and $\tau_{-}$. It is to be noted that, since the transformation removed odd terms of a given order because of the properties of the commutators of odd terms with the $(m \beta)$ term, each transformation will be of one higher order in reciprocal masses than the order of the term to be removed; hence, odd terms of the order $\left(1 / m^{2}\right)$ or greater can always be removed without making further contribution and will consequently be ignored as they arise.

Evaluating the commutators indicated in (7) and collecting terms, one finds

$$
\begin{aligned}
\mathcal{H}^{\prime}=\mathcal{H}_{1} & +\mathcal{H}_{2}+\left(\frac{f}{2 m}-\frac{g}{\mu}\right) \Psi^{*} \boldsymbol{\sigma} \cdot \nabla\left(\tau_{-} \phi+\tau_{+} \phi^{*}\right) \Psi \\
& +\frac{1}{2 m} \Psi^{*} \beta p^{2} \Psi+\frac{f^{2}}{2 m} \Psi^{*} \beta \phi^{*} \phi \Psi \\
& -\left(\frac{i f^{2}}{8 m^{2}}-\frac{i g f}{2 \mu m}\right) \Psi^{*} \tau_{\boldsymbol{z}}\left(\pi^{*} \phi^{*}-\pi \phi\right) \Psi \\
& +\left[\frac{1}{2}\left(\frac{g}{\mu}\right)^{2}-\frac{g f}{4 \mu m}+\frac{f^{2}}{8 m^{2}}\right](\mathrm{DI}) \\
& +\frac{g f}{4 \mu m}\left[\Psi^{*} \beta \gamma^{5} \tau_{+} \Psi \Psi^{*} \beta \gamma^{5} \tau_{-} \Psi\right. \\
& \left.+\Psi^{*} \beta \gamma^{5} \tau_{-} \Psi \Psi^{*} \beta \gamma^{5} \tau_{+} \Psi\right]+\left(\frac{f}{8 m^{2}}-\frac{\dot{g}}{2 \mu m}\right) \\
& \times \Psi^{*} \beta \boldsymbol{\sigma} \cdot\left[\mathbf{p}\left(\tau_{-} \pi^{*}+\tau_{+} \pi\right)+\left(\tau_{-} \pi^{*}+\tau_{+} \pi\right) \mathbf{p}\right] \Psi \\
& -\frac{f}{2 m} \Psi^{*} \gamma^{5}\left(\tau_{-} \pi^{*}+\tau_{+} \pi\right) \Psi
\end{aligned}
$$




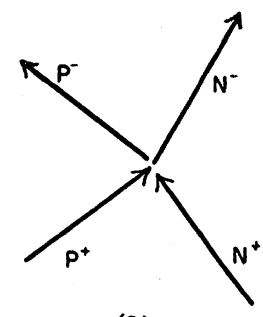

(a)

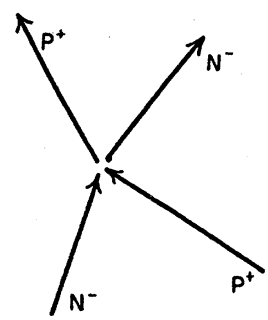

(b)

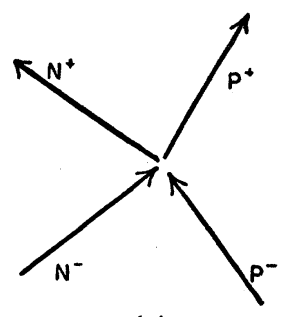

(a')

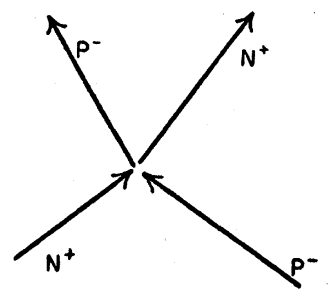

(b)
FIG. 1. Diagrams of the processes represented by the direct interaction terms.

The last term is odd and of the order $(1 / M)$. However the transformation required to remove it will be of the order $\left(1 / M^{2}\right)$, and the only commutator that it will have with $\mathcal{F}^{\prime}$ that will be proportional to $\left(1 / M^{2}\right)$ will be the one with $\mathfrak{H C}_{2}$. But this commutator will be odd; hence, the last term may now simply be dropped. The term involving the gradient of the meson momenta is also ignorable to this order. This is explicitly justified by noting that the term in question is, to the order $\left(1 / m^{2}\right)$, simply the commutator of

$$
\begin{aligned}
& G=-\left(\frac{f}{8 m^{2}}-\frac{g}{2 \mu m}\right) \int \Psi^{*} \beta \boldsymbol{\sigma} \cdot {\left[\mathbf{p}\left(\tau_{-} \phi+\tau_{+} \phi^{*}\right)\right.} \\
&\left.+\left(\tau_{-} \phi+\tau_{+} \phi^{*}\right) \mathbf{p}\right] \Psi d^{3} x
\end{aligned}
$$

with $\mathcal{H}^{\prime}$, and hence is to this order simply $\partial G / \partial t$ $=i\left[\mathcal{H C}^{\prime}, G\right]$. As a time derivative, it should have no diagonal matrix elements and therefore should be removable from this order by a canonical transformation. That this is true is obvious, and the transformation required is simply $\exp (-i G)$.

Consideration must now be given to the direct interaction terms in the Hamiltonian which, in the form in which they are exhibited, are neither even nor removable by canonical transformations. The terms to which attention shall be given are of the form

and

$$
\Psi^{*} \gamma^{5} \tau_{-} \Psi \Psi^{*} \gamma^{5} \tau_{+} \Psi
$$

$$
\Psi^{*} \beta \gamma^{5} \tau_{-} \Psi \Psi^{*} \beta \gamma^{5} \tau_{+} \Psi \text {. }
$$

If one rewrites the first of these in the form

$$
\begin{aligned}
& \Psi^{*} \Gamma_{1} \tau_{-} \Psi \Psi^{*} \Gamma_{2} \tau_{+} \Psi+\Psi^{*} \Gamma_{2} \tau_{-} \Psi \Psi^{*} \Gamma_{1} \tau_{+} \Psi \\
& \text { where } \\
& +\Psi^{*} \Gamma_{1} \tau_{-} \Psi \Psi^{*} \Gamma_{1} \tau_{+} \Psi+\Psi^{*} \Gamma_{2} \tau_{-} \Psi \Psi^{*} \Gamma_{2} \tau_{+} \Psi, \\
& \Gamma_{1}=\left(\frac{1+\beta}{2}\right) \gamma^{5}\left(\frac{1-\beta}{2}\right), \quad \Gamma_{2}=\left(\frac{1-\beta}{2}\right) \gamma^{5}\left(\frac{1+\beta}{2}\right),
\end{aligned}
$$

(the second term reducing to exactly the same form except that the unsymmetric terms are negative), one observes that there is a fundamental difference between the first two components which are unsymmetric in the $\Gamma$ 's and the second two symmetric components. The unsymmetric terms accomplish transitions during which there is no apparent change in the number of either positive or negative energy particles in the field, whereas the transitions resulting from the symmetric type terms do correspond to changes in the number of such particles. The nature of the difference implied here becomes clear immediately upon inspection of schematic diagrams representing the processes involved (Fig. 1).

Diagrams $a$ and $a^{\prime}$ represent the processes accomplished by the symmetric terms in which the number of positive energy particles $\left(P^{+}, N^{+}\right)$and the number of negative energy particles $\left(P^{-}, N^{-}\right)$changes in the course of the interaction. However, the processes schematized in $b$ and $b^{\prime}$ do not involve any apparent changes in the number of positive and/or negative energy particles.

Now, transitions involving such changes in the number of particles in the field require interaction energies at least of the order of the rest energies of the particles, and hence, correspond to fundamentally relativistic processes. Consequently, the symmetric components of the direct interaction terms should be removable from the Hamiltonian by means of a canonical transformation without generating any further contributions to the Hamiltonian of the same order as the terms themselves. This is indeed the case for

$$
\begin{aligned}
{\left[\int \Psi^{*} \Gamma_{1} \tau_{-} \Psi \Psi^{*} \Gamma_{1} \tau_{+} \Psi d^{3} x, \Psi^{*} \beta m \Psi\right] } & \\
& =-2 m \Psi^{*} \Gamma_{1} \tau_{-} \Psi \Psi^{*} \Gamma_{1} \tau_{+} \Psi .
\end{aligned}
$$

Thus, if one chooses a canonical transformation whose generating function is

$$
-(i / 2 m) \int \Psi^{*} \Gamma_{1} \tau_{-} \Psi \Psi^{*} \Gamma_{1} \tau_{+} \Psi d^{3} x
$$

the corresponding symmetric term will be canceled from JC. It must be pointed out here that the removal of the relativistic components of the direct interactions does not in any way depend upon the assumption of equal masses for the interacting particles.

Conversely, the unsymmetric terms represent scattering in which the energy exchange may very well be small. Thus these terms should be even according to the criterion of commutativity with the $\beta m$ term, as they are from the physical criterion that there is no observable coupling of positive and negative energy states. This one easily verifies by direct calculation.

The even character of the terms, as well as their physical significance, becomes still more obvious in the light of the following argument. Making use of the 
relation

$$
\begin{aligned}
& \int \Psi_{r}{ }^{*} B_{r s} \Psi_{s} \Psi_{i}^{*} A_{i j} \Psi_{j} d^{3} x \\
& =-\int \Psi_{r}^{*} \Psi_{i}{ }^{*} B_{r s} A_{i j} \Psi_{s} \Psi_{j} d^{3} x+\iint \Psi_{r}^{*}\left(x^{\prime}\right) \\
& \times B_{r s} A_{s j} \Psi_{j}(x) \delta\left(\mathbf{x}-\mathbf{x}^{\prime}\right) \delta\left(\mathbf{x}-\mathbf{x}^{\prime}\right) d^{3} x d^{3} x^{\prime},
\end{aligned}
$$

one easily deduces that

$$
\begin{aligned}
& {\left[\Psi^{*} \Gamma_{1} \tau_{-} \Psi \Psi^{*} \Gamma_{2} \tau_{+} \Psi+\Psi^{*} \Gamma_{2} \tau_{+} \Psi \Psi^{*} \Gamma_{1} \tau_{-} \Psi\right.} \\
& \left.+\Psi^{*} \Gamma_{2} \tau_{-} \Psi \Psi^{*} \Gamma_{1} \tau_{+} \Psi+\Psi^{*} \Gamma_{1} \tau_{+} \Psi \Psi^{*} \Gamma_{2} \tau_{-} \Psi\right] d^{3} x \\
& =\iint \Psi^{*}\left(x^{\prime}\right) \Psi(x) \delta\left(\mathbf{x}-\mathbf{x}^{\prime}\right) \delta\left(\mathbf{x}-\mathbf{x}^{\prime}\right) d^{3} x d^{3} x^{\prime} \\
& \quad-2 \int \Psi_{r}^{*} \Psi_{i}^{*}\left(\Gamma_{1} \tau_{-}\right)_{r s}\left(\Gamma_{2} \tau_{+}\right)_{i j} \Psi_{s} \Psi_{j} d^{3} x \\
& -2 \int \Psi_{r}^{*} \Psi_{i}^{*}\left(\Gamma_{2} \tau_{-}\right)_{r s}\left(\Gamma_{1} \tau_{+}\right)_{i j} \Psi_{s} \Psi_{j} d^{3} x
\end{aligned}
$$

The first (infinite) term represents a self-energy contribution from the interaction, which presumably is removable by an appropriate renormalization of the original Hamiltonian. The second two terms may be still further clarified by transforming to momentumspace and noting that in the eight-dimensional matrix representation,

$$
\begin{aligned}
& \Gamma_{1} \tau_{-}=\left(\begin{array}{llll}
0 & 0 & 0 & 0 \\
0 & 0 & 0 & 0 \\
0 & I & 0 & 0 \\
0 & 0 & 0 & 0
\end{array}\right), \quad \Gamma_{2} \tau_{+}=\left(\begin{array}{llll}
0 & 0 & 0 & 0 \\
0 & 0 & I & 0 \\
0 & 0 & 0 & 0 \\
0 & 0 & 0 & 0
\end{array}\right), \\
& \Gamma_{1} \tau_{+}=\left(\begin{array}{llll}
0 & 0 & 0 & I \\
0 & 0 & 0 & 0 \\
0 & 0 & 0 & 0 \\
0 & 0 & 0 & 0
\end{array}\right), \quad \Gamma_{2} \tau_{-}=\left(\begin{array}{llll}
0 & 0 & 0 & 0 \\
0 & 0 & 0 & 0 \\
0 & 0 & 0 & 0 \\
I & 0 & 0 & 0
\end{array}\right),
\end{aligned}
$$

where $I$ is the two-by-two unit matrix. One obtains (ignoring the infinite term)

$$
\begin{aligned}
& -\frac{2}{(2 \pi)^{6}} \sum_{s^{\prime \prime \prime} s^{\prime \prime} s^{\prime} s} \int d^{3} x d^{3} p^{\prime \prime \prime} d^{3} p^{\prime \prime} d^{3} p^{\prime} d^{3} p \\
& \times \exp \left[-i \mathbf{x} \cdot\left(\mathbf{p}^{\prime \prime \prime}+\mathbf{p}^{\prime \prime}-\mathbf{p}^{\prime}-\mathbf{p}\right)\right] \\
& \times a_{p^{\prime \prime \prime} s^{\prime \prime}} * a_{p^{\prime \prime} s^{\prime \prime}} * a_{p^{\prime} s^{\prime}} a_{p s} \\
& \times u^{*}\left(p^{\prime \prime \prime}, s_{5,6}{ }^{\prime \prime \prime}\right) I u\left(p^{\prime}, s_{3,4}{ }^{\prime}\right) \\
& \times u^{*}\left(p^{\prime \prime}, s_{3,4}{ }^{\prime \prime}\right) I u\left(p, s_{5,6}\right), \\
& -\frac{2}{(2 \pi)^{6}} \sum_{s^{\prime \prime \prime} s^{\prime \prime} s^{\prime} s} \int d^{3} x d^{3} p^{\prime \prime \prime} d^{3} p^{\prime \prime} d^{3} p^{\prime} d^{3} p \\
& \times \exp \left[-\left(i \mathbf{x} \cdot\left(\mathbf{p}^{\prime \prime \prime}+\mathbf{p}^{\prime \prime}-\mathbf{p}^{\prime}-\mathbf{p}\right)\right]\right. \\
& \times a_{p^{\prime \prime \prime} s^{\prime \prime \prime}}{ }^{*} a_{p^{\prime \prime} s^{\prime \prime}} * a_{p^{\prime} s^{\prime}} a_{p_{s}} u^{*}\left(p^{\prime \prime \prime}, s_{7,8}{ }^{\prime \prime \prime}\right) \\
& \times I u\left(p^{\prime}, s_{1,2}\right) u^{*}\left(p^{\prime \prime}, s_{1,2}{ }^{\prime \prime}\right) I u\left(p, s_{7,8}\right),
\end{aligned}
$$

where

$$
\begin{aligned}
& u\left(p, s_{1,2}\right) \rightarrow \text { positive energy neutron, spin }\left\{\begin{array}{l}
\text { up } \\
\text { down }
\end{array}\right\}, \\
& u\left(p, s_{3,4}\right) \rightarrow \text { negative energy neutron, spin }\left\{\begin{array}{l}
\text { up } \\
\text { down }
\end{array}\right\}, \\
& u\left(p, s_{5,6}\right) \rightarrow \text { positive energy proton, } \operatorname{spin}\left\{\begin{array}{l}
\text { up } \\
\text { down }
\end{array}\right\}, \\
& u\left(p, s_{7,8}\right) \rightarrow \text { negative energy proton, } \operatorname{spin}\left\{\begin{array}{l}
\text { up } \\
\text { down }
\end{array}\right\} .
\end{aligned}
$$

It is to be noted that $\gamma^{5}$ behaves like a unit matrix in the two-dimensional spin sub-space, and therefore the interaction, in this case, does not involve spin transitions.

If the even, nonremovable part of the direct interaction be designated as ( $E$ of DI), the odd parts removed by canonical transformations, and the terms combined, keeping in mind that the even parts of the terms of the form

$$
\Psi^{*} \beta \gamma^{5} \tau_{-} \Psi \Psi^{*} \beta \gamma^{5} \tau_{+} \Psi
$$

are of exactly the same form as those of the term types just discussed but opposite in sign, then the Hamiltonian density becomes

$$
\begin{aligned}
\mathcal{F}=\pi^{*} \pi & +\nabla \phi^{*} \cdot \nabla \phi+u^{2} \phi^{*} \phi+\Psi^{*} \beta m \Psi \\
& +\frac{1}{2 m} \Psi^{*} \beta p^{2} \Psi+\left(\frac{f}{2 m}-\frac{g}{\mu}\right) \Psi^{*} \boldsymbol{\sigma} \cdot \nabla\left(\tau_{-} \phi+\tau_{+} \phi^{*}\right) \Psi \\
& +\frac{f^{2}}{2 m} \Psi^{*} \beta \phi^{*} \phi \Psi \\
& -\left(\frac{i f^{2}}{8 m^{2}}-\frac{i g f}{2 \mu m}\right) \Psi^{*} \tau_{z}\left(\pi^{*} \phi^{*}-\pi \phi\right) \Psi \\
& +\left[\frac{1}{2}\left(\frac{g}{\mu}\right)^{2}-\frac{g f}{2 \mu m}+\frac{f^{2}}{8 m^{2}}\right](E \text { of } \mathrm{DI}) .
\end{aligned}
$$

Note that the Hamiltonian is now in a form such that, if the eight-component wave operators $\Psi$ are replaced by two sets of four-component operators $\frac{1}{2}(1+\beta) \Psi$ and $\frac{1}{2}(1-\beta) \Psi$, it breaks up into a sum of essentially three different sets of terms. Two of these sets, resulting from the decomposition of those terms which are independent of the odd isotopic spin operators $\tau_{-}$and $\tau_{+}$contain creation and destruction operators for either positive energy states of both protons and neutrons or negative energy states. The third set of terms is comprised of those which are odd in isotopic spin space; namely the terms resulting from the reduction to four-component description of the term $\Psi^{*} \cdot \boldsymbol{\nabla}\left(\tau_{-} \phi+\tau_{+} \phi^{*}\right) \Psi$ and the even parts of the direct 
interaction terms. Note that in the former the coupling is strictly between different charge states of the same energy character only, thus these reduced terms separate in the four-component description; whereas the latter do not separate-both sets of four-component wave operators are essential to the formulation of these terms -in spite of the fact that the coupling between nucleoonic states of different energy character is observationally unreal.

It should be pointed out here that this represents a nonrelativistic statement of an "equivalence theorem" for pseudoscalar and pseudovector couplings in the pseudoscalar meson theory. ${ }^{4}$ Such equivalence theorems have already been derived ${ }^{5}$ for the case of a given external meson field. To the first order in the coupling constant it is seen that the nonrelativistic Hamiltonians for either pseudoscalar coupling or pseudovector coupling or both are completely equivalent, assuming an appropriate choice of the coupling constant. To the second order these three different Hamiltonians are equivalent only to within certain additive terms.

\section{Similar Particles}

One other case of interest should be mentioned here; namely, that of the interaction of two similar particles through the intermediation of a second field. As an example, the interactions of similar charged particles via the electromagnetic field will be considered. The question that arises is again relative to the removability of contact, or direct interaction, terms. The reason that the issue here requires some special attention is that, in this instance, there is only one Dirac field involved and hence formally the situation appears to differ considerably from the case previously discussed. However, as will be seen, this case is strictly analogous to the previous one, physically, and the decomposition of the direct interaction terms into odd, relativistic components and even, nonrelativistic components, and the removal of the odd components proceeds exactly as before.

Since it is of interest here merely to demonstrate the generation of "odd-odd" terms and discuss their reduction to nonrelativistic form, it is convenient for the sake of simplicity, and quite sufficient, to restrict ourselves to the particular case of the interaction of charged particles via the transverse electromagnetic field, omitting Coulomb interactions. The Hamiltonian may then be written as

$$
\mathfrak{F}=\mathfrak{H}_{e m}+\psi^{*} \beta m \psi+\psi^{*} \boldsymbol{\alpha} \cdot(\mathbf{p}-e \mathbf{A}) \psi,
$$

${ }^{4}$ K. M. Case, Phys. Rev. 76, 14 (1949). Note that the statement of the equivalence theorem obtained herein is not in agreement with the one derived by Case. The disagreement disappears if one chooses Case's relativistic statement of the theorem after altering the term $(2 g / \hbar c k)\left(f-2 k_{0} g / k\right) \phi^{*} \phi \bar{\psi} \psi$ to $\mathrm{read}(2 g / \hbar c k)\left(f-k_{0} g / k\right)$ $\times \phi^{*} \phi \bar{\psi} \psi$ and then reduces the altered interaction Hamiltonian to nonrelativistic form.

${ }^{5}$ M. Sugarawara and Y. Ono, Prog. Theoret. Phys. 5, 901 (1950). where

$$
\begin{gathered}
\mathcal{C}_{e m}=\frac{1}{2}\left[|\mathbf{P}|^{2}+|\nabla \times \mathbf{A}|^{2}\right], \\
{\left[A_{i}(x), P_{j}\left(x^{\prime}\right)\right]_{-}=i \delta_{i j} \delta\left(\mathbf{x}-\mathbf{x}^{\prime}\right)}
\end{gathered}
$$

and $\mathbf{A}$ and $\mathbf{P}$ satisfy the operator equations

$$
(\boldsymbol{\nabla} \cdot \mathbf{A}) \Phi=0, \quad(\boldsymbol{\nabla} \cdot \mathbf{P}) \Phi=0,
$$

$\Phi$ being a state vector for the system.

If now one chooses a canonical transformation $e^{i T}$, where

$$
T=-(i / 2 m) \int \psi^{*} \beta \boldsymbol{\alpha} \cdot(\mathbf{p}-e \mathbf{A}) \psi d^{3} x,
$$

then

$$
\mathcal{F C}^{\prime}=e^{i T} \mathcal{H} e^{-i T}
$$

will be free of odd operators to the order zero in $(1 / m)$ and will contain as a consequence of $\left[T, \mathfrak{H}_{e m}\right]^{2}$ terms of the form $\psi^{*} \beta \boldsymbol{\alpha} \psi \cdot \psi^{*} \beta \boldsymbol{\alpha} \psi$ which are of the second order in $(1 / m)$. This is of the type with which we are here concerned.

This term may be written as

$$
\begin{aligned}
& \psi^{*} \boldsymbol{\Gamma}_{1} \psi \cdot \psi^{*} \boldsymbol{\Gamma}_{1} \psi+\psi^{*} \boldsymbol{\Gamma}_{2} \psi \cdot \psi^{*} \boldsymbol{\Gamma}_{2} \psi \\
&-\psi^{*} \boldsymbol{\Gamma}_{1} \psi \cdot \psi^{*} \boldsymbol{\Gamma}_{2} \psi-\psi^{*} \boldsymbol{\Gamma}_{2} \psi \cdot \psi^{*} \boldsymbol{\Gamma}_{1} \psi,
\end{aligned}
$$

where

$$
\Gamma_{1}=\left(\frac{1+\beta}{2}\right) \alpha\left(\frac{1-\beta}{2}\right)
$$

and

$$
\boldsymbol{\Gamma}_{2}=\left(\frac{1-\beta}{2}\right) \alpha\left(\frac{1+\beta}{2}\right) .
$$

The first two symmetric components are again odd and essentially relativistic; i.e., they represent the scattering of two positive energy particles (or two negative energy particles) into negative (or positive) energy states. Therefore, these terms should be and are removable for

$$
\left[\lambda \int \psi^{*} \boldsymbol{\Gamma}_{1} \psi \cdot \psi^{*} \boldsymbol{\Gamma}_{1} \psi d^{3} x, \psi^{*} \beta m \psi\right]=-4 m \lambda \psi^{*} \boldsymbol{\Gamma}_{1} \psi \cdot \psi^{*} \boldsymbol{\Gamma}_{1} \psi
$$

and similarly for the other odd term. Thus, by an appropriate choice of $\lambda$, the canonical transformation whose generating function is $\lambda S \psi^{*} \boldsymbol{\Gamma}_{1} \psi \cdot \psi^{*} \boldsymbol{\Gamma}_{1} \psi d^{3} x$ will remove the odd components of the "odd-odd" terms.

The second two unsymmetric terms are even and essentially nonrelativistic in character, for they represent the scattering of one positive and one negative energy particle into negative and positive energy states, respectively. Thus, so far as an observer is concerned, these terms simply accomplish the scattering of electrons by positrons. The even character of these can readily be verified by straightforward calculation of their commutators with the $\beta m$ term. If these terms are combined and then separated explicitly into their infinite and finite components, and the finite com- 
ponents transformed into momentum-space and reduced to a description in spin sub-space as was done in the previous example, it will be seen that these even parts of the direct interaction terms accomplish spin transitions. ${ }^{6}$

${ }^{6}$ A related paper by S. Tani, Prog. Theoret. Phys. 6, 267 (1951), has recently been published. In this paper, Tani employs the
The writer gratefully acknowledges his indebtedness to Dr. Leslie L. Foldy for suggesting this problem, and for his invaluable advice and criticism throughout the course of the work.

transformation for a detailed elucidation of the physical significance of the operators commonly occurring in theories of fermions interacting with fields in the single particle formalism.

\title{
Radioisotopes of Bromine
}

\author{
S. C. Fultz* AND M. L. Poor \\ Ohio State University, Columbus, Ohio \\ (Received December 31, 1951)
}

\begin{abstract}
Selenium metal enriched with $\mathrm{Se}^{76}$ was bombarded with 7.3-Mev protons. A new 17.2-hour activity in bromine was observed and is assigned to $\mathrm{Br}^{76}$. The radiations of $\mathrm{Br}^{83}, \mathrm{Br}^{80}, \mathrm{Br}^{77}, \mathrm{Br}^{76}$, and $\mathrm{Br}^{75}$ have been examined by use of a $180^{\circ}$ focusing spectrometer and coincidence methods. $\mathrm{Br}^{83}$ emits a simple negatron spectrum with a maximum energy of $0.94 \pm 0.02 \mathrm{Mev}$. The ground state of $\mathrm{Br}^{80}$ emits a complex negatron spectrum with components: $1.97 \pm 0.03 \mathrm{Mev}$ ( 80 percent), $1.1 \mathrm{Mev}$ (11 percent), $0.7 \mathrm{Mev}$ (9 percent). The gamma-rays of $\mathrm{Br}^{77}$ are associated with the $K$-capture process. The ground state of $\mathrm{Br}^{76}$ emits positrons having a complex spectrum with components $3.57 \pm 0.07 \mathrm{Mev}$ (46 percent), $1.7 \mathrm{Mev}$ (10 percent), $1.1 \mathrm{Mev}$ (11 percent), $0.8 \mathrm{Mev}$ (14 percent), $0.6 \mathrm{Mev}$ (19 percent). Gamma-rays identified with $\mathrm{Br}^{76}$ have energies of $0.25,0.33,0.37,0.42,0.68,0.75,0.96$, and $1.2 \mathrm{Mev}$. The ground state of $\mathrm{Br}^{75}$ decays by emission of positrons having a complex spectrum with components $1.70 \pm 0.02 \mathrm{Mev}$ (46 percent), $0.8 \mathrm{Mev}$ (20 percent), 0.6 Mev (15 percent), 0.3 Mev (19 percent).
\end{abstract}

\section{INTRODUCTION}

$\mathbf{R}^{\mathrm{s}}$ ADIATIONS from several radioisotopes of bromine have been studied by use of the beta-ray spectrometer and by absorption and coincidence measurements. The radioisotopes were obtained by proton and deuteron bombardments on selenium metal. Decay of their activities was followed with the aid of a Wulf electrometer filled with freon to a pressure of about 2 atmospheres. A magnetic field was used to separate positrons and negatrons, thus enabling decays of the activities giving rise to these particles to be determined separately by use of a G-M counter.

The spectrometer employed was of the $180^{\circ}$ focusing type with a trajectory radius of $16 \mathrm{~cm}$. The slits were made long in the direction of the magnetic field in order to obtain maximum possible intensity ${ }^{1}$ for a predetermined resolution of about two percent. The spectrometer source consisted of fine particles of activated selenium metal evenly spread on a thin ribbon of Zapon and held in place by a thin layer of collodion. The density of the source and backing was about $10 \mathrm{mg} / \mathrm{cm}^{2}$. The thickness of the G-M counter window was 2.5 $\mathrm{mg} / \mathrm{cm}^{2}$.

\section{THE 17.2-HOUR $\mathrm{Br}^{76}$}

Two types of samples of selenium metal were bombarded with protons of 7.3-Mev energy. One type con-

\footnotetext{
* Fellow, National Cancer Institute of the National Institutes of Health, Public Health Service, Federal Security Agency, Bethesda, Maryland.

${ }^{1}$ G. E. Owen, Rev. Sci. Instr. 20, 916 (1949).
}

tained the natural isotopic mixture of stable selenium isotopes, while the other type contained $\mathrm{Se}^{76}$ enriched from 9.0 to 41.5 percent. In addition to the well-known bromine activities of 4.4-hour $\mathrm{Br}^{80}, 36$-hour $\mathrm{Br}^{82}$, and 2.4-day $\mathrm{Br}^{77}$, a new activity of 17.2 hours was observed. Some evidence for the existence of a 15.7-hour activity had been observed previously ${ }^{2}$ and early results for the work discussed below have already been reported. ${ }^{3}$ Recently J. Hollander of the University of California privately communicated that a 16-hour bromine activity has been obtained by bombardment of arsenic with alpha-particles.

In Fig. 1 are shown decay curves obtained for the total activity and for the electromagnetically separated particles emitted from a Se ${ }^{76}$ enriched sample of selenium metal which was bombarded with protons of $7.3-\mathrm{Mev}$ energy. Five components are present in the decay curve for the total activity. Their relative contributions to the total activity at the beginning of the measurements can be read from the intercepts of the straight lines. Similar sets of curves were obtained for samples of natural selenium bombarded in the same manner.

In Table I is given activity data obtained from three bombardments. The bottom row contains activities obtained when the bromine was chemically separated from the selenium metal. The activity values listed have been adjusted to take into account differences in

${ }^{2}$ G. T. Seaborg and I. Perlman, Revs. Modern Phys. 20, 585 (1948).

${ }^{3}$ S. C. Fultz and M. L. Pool, Phys. Rev. 83, 875 (1951). 